\title{
Optimal Portfolio and Background Risk: An Exact and an Approximated Solution*
}

\author{
Francesco Menoncin ${ }^{a, \dagger}$ \\ ${ }^{a}$ IRES, Université catholique de Louvain, Louvain-La-Neuve, Belgium
}

March 17, 2002

\begin{abstract}
This paper analyses the portfolio problem of an investor who wants to maximize the expected exponential utility of his terminal wealth both in a complete and an incomplete financial market. The investor must cope with a set of stochastic investment opportunities and two sets of background risks. If the market is complete we are able to find an exact solution. Instead, if the market is incomplete, we suggest an approximated general solution. Contrary to other exact solutions obtained in the literature, all our results are obtained without specifying any particular functional form for the stochastic variables involved in the problem.
\end{abstract}

JEL classification: G11, C61.

Key words: asset allocation, background risk, Feynman-Kac theorem, stochastic investment opportunities

${ }^{*}$ We have received helpful comments from R. Anderson, G. Deelstra, O. Scaillet and D. Sciutti. We gratefully acknowledge financial support from the Belgian French Community's program "Action de Recherche Concentrée" 99/04-235.

†IRES, Université catholique de Louvain, Place Montesquieu, 3, 1348 - Louvain-La-Neuve, Belgium. Tel: 0032-10-474035; fax: 0032-10-473945; e-mail: menoncin@ires.ucl.ac.be 


\section{Introduction}

This work analyses the issue of optimal portfolio policy in a multi-period model where investors maximize the expected utility of their terminal wealth facing, in particular, some risks outside the financial market that we will refer to as "background risks". Furthermore, our work offers a contribution to the investment problem in the rather general case where the value of assets depends on the stochastic behaviour of a set of state variables.

The vector of state variables contains all the stochastic variables directly affecting the asset prices but indirectly affecting the investors' wealth. For a review of all variables which can affect the asset prices readers are referred to Campbell (2000) who offers a survey of the most important contributions in this field.

Besides these state variables, we consider two sets of stochastic processes, called in the paper "background variables". One set of background variables affects the level of investor's wealth (and we will call it level background risk) while the other set affects the growth rate of investor's wealth (and we will call it ratio background risk). In the first set we can find variables such as the investor's wages (see for instance Franke, Peterson, and Stapleton, 2001), while in the second set we can find variables such as the inflation rate or the exchange rate (in the case of foreign investments).

This characteristic makes our model quite general because it can be applied, for example, to pension funds (see for instance Blake, 1998, Blake, Cairns, and Dowd, 1998, and Boulier, Huang, and Taillard, 2001) or to insurance companies (see Young and Zariphopoulou, 2000). A lot of attempts have been made in the literature for introducing the background risk in the optimal portfolio problem (see the works above mentioned) but often the authors make a lot of strong assumptions in order to reach a framework which can be handled. In this work, the presence of the ratio background risk can solve some of the problem which are generally faced in this type of models. In particular, we do not use the most common approximation for measuring the wealth growth rate net of the ratio background risk, but we use the exact formula. This allows us to solve some technicalities which make the problem very difficult to solve when the usual approximation is considered.

In this paper we follow the traditional route to use the stochastic dynamic programming technique (Merton, 1969, 1971) leading to the Hamilton-JacobiBellman (HJB) equation. " For the method called "martingale approach" the reader is referred to Cox and Huang (1989, 1991), and Lioui and Poncet (2000). We just underline that in this work we are able to reach the same qualitative results as Lioui and Poncet even if they do not consider any background risk.

In our framework, we find that the optimal portfolio is formed by three components: (i) a preference free component minimizing the investor's wealth diffusion variance and immunizing the investor's portfolio against the background risks, (ii) a speculative part proportional to both the Sharpe ratio of

\footnotetext{
${ }^{1} \varnothing \mathrm{ksendal}(2000)$ and Björk (1998) offer a complete derivation of the HJB equation.
} 
investor's portfolio and the inverse of the Arrow-Pratt risk aversion index, and (iii) a component depending on the derivatives of the value function (indirect utility function) with respect to all the state and background variables. The last component is the only one depending on the investor's time horizon.

In order to find the explicit solution for the value function, it is necessary to solve the HJB equation. Unfortunately, solving this highly non-linear PDE is the most difficult task of the stochastic optimal control approach. In fact, some algebraic solutions can only be obtained in very special cases. In particular, we refer to the works of Kim and Omberg (1996), Wachter (1998), Boulier, Huang, and Taillard (2001), and Deelstra, Grasselli, and Koehl (2001).

In the present work we show that if the financial market is complete, then we can find an exact solution to our optimal portfolio problem with background risk. This result can be obtained thanks to the insertion of the ratio background risk affecting the growth rate of investor's wealth (namely the inflation risk). In fact, if this kind of risk exists, then the riskless asset is no more "riskless". In particular, if the investor wants to maximize the expected value of his terminal real wealth, then for him no riskless asset exists because the nominal riskless asset does not cover the portfolio against the inflation risk. Furthermore, we outline that, contrary to the exact solutions above mentioned, we do not specify any particular functional form for the behaviour of the stochastic variables involved in the problem and we reach, in this way, a very general solution indeed.

If the completeness hypothesis does not hold, then we propose a general approximated solution to the HJB equation. In particular, our work concentrates on the hypothesis that the value function has a suitable form under which the Feynman-Kac theorem can be applied to the HJB equation. Thus, even if in an incomplete market our solution is exact only under particular conditions that must hold on the value function, we find that it stays valid as an approximated solution under conditions which are not very restrictive.

We underline that the exact solutions presented in Kim and Omberg (1996), Boulier, Huang, and Taillard (2001), and Deelstra, Grasselli, and Koehl (2001), consider only one state variable and do not take into account any background risk. Instead, our model is able to determine both an exact and an approximated solution when there exists a set of generic state and background variables. Thus, our framework seems to be very general and able to be applied to many particular cases.

Through this work we consider agents trading continuously in a frictionless, arbitrage-free market until time $H$, which is the horizon of the economy. Furthermore, we analyse both a complete and an incomplete market structure.

The paper is structured as follows. Section 2 details the general economic framework and exposes the stochastic differential equations describing the behaviour of asset prices, state variables and background variables. In Section 3 the optimal portfolio composition is computed. This section presents our main results: an exact solution of the problem if the financial market is complete, and an approximated solution when the market is not complete. Section 4 concludes. 


\section{The economy}

In this paper we consider an economy where the behaviour of asset prices is described by stochastic differential equations. Furthermore, the volatility and drift coefficients of these equations depend on a set of state variables which are represented by diffusion processes.

Before specifying the market structure, here we recall the fundamental results about the market arbitrage and completeness. We define as $\{S(t, \omega)\}_{t \in\left[t_{0}, H\right]}$ a market where there are $n$ risky assets and one riskless asset $(G)$ whose nominal prices follow the stochastic differential equations:

$$
\begin{aligned}
d G(t, \omega) & =r(t, \omega) G d t, \\
G\left(t_{0}\right) & =G_{0}, \\
d S(t, \omega) & =\mu \underset{n \times 1}{(t, \omega) d t+\Sigma^{\prime}(t, \omega) \underset{n \times k}{n \times 1} \underset{k \times 1}{d},}, \\
S\left(t_{0}\right) & =S_{0},
\end{aligned}
$$

where $d W$ is the differential of a $k$-dimensional Wiener process, $r(t, \omega)$ is the instantaneous riskless interest rate, $\omega \in \Theta$ and $\Theta$ is the set from which we define the $\sigma$-algebra through which the Wiener processes are measured. Hereafter, the prime denotes transposition. We say that the market $\{S(t, \omega)\}_{t \in\left[t_{0}, H\right]}$ is normalized if $G(t, \omega) \equiv 1$. This hypothesis means that the riskless asset is the numeraire of the economy. Any market can always be normalized by putting $\bar{S}(t, \omega)=G(t, \omega)^{-1} S(t, \omega)$.

We present the main results concerning completeness and arbitrage in this kind of market (for the proofs of the two following theorems see Øksendal, 2000).

Theorem 1 A market $\{S(t, \omega)\}_{t \in\left[t_{0}, H\right]}$ is arbitrage free if and only if there exists a $k$-dimensional vector $\xi(t, \omega)$ such that:

$$
\Sigma(t, \omega)^{\prime} \xi(t, \omega)=\mu(t, \omega)-r(t, \omega) S(t, \omega),
$$

and such that:

$$
\mathbb{E}\left[e^{\frac{1}{2} \int_{t_{0}}^{H}\|\xi(t, \omega)\|^{2} d t}\right]<\infty
$$

Theorem 2 A market $\{S(t, \omega)\}_{t \in\left[t_{0}, H\right]}$ is complete if and only if there exists a unique $k$-dimensional vector $\xi(t, \omega)$ such that:

$$
\Sigma(t, \omega)^{\prime} \xi(t, \omega)=\mu(t, \omega)-r(t, \omega) S(t, \omega),
$$

and such that:

$$
\mathbb{E}\left[e^{\frac{1}{2} \int_{t_{0}}^{H}\|\xi(t, \omega)\|^{2} d t}\right]<\infty
$$


If on the market there are less assets than risk sources $(n<k)$, then the market cannot be complete even if it is arbitrage free. In this work we assume that $n \leq k$ and that the rank of matrix $\Sigma$ is maximum (i.e. it equals $n$ ). We will analyse both cases in which the market is incomplete $(n<k)$ and complete $(n=k)$.

\subsection{The market structure}

We suppose that asset prices are affected by a set of stochastic state variables. For a review of all variables which can affect the asset prices readers are referred to Campbell (2000) who offers a survey of the most important contributions in this field.

In this paper we suppose that these risk sources follow the stochastic differential equation:

$$
\begin{aligned}
\underset{s \times 1}{X X} & =\underset{s \times 1}{(X, t) d t}+\underset{s \times k}{(\underset{x}{X}, t)^{\prime}} \underset{k \times 1}{W}, \\
X\left(t_{0}\right) & =X_{0},
\end{aligned}
$$

where $s$ is the number of state variables and $d W$ is the differential of a $k$-dimensional Wiener process whose components are independent. ${ }^{2}$

Given these variables we can write the process describing the behaviour of asset prices like the following stochastic differential equation:

$$
\begin{aligned}
& \underset{n \times 1}{d S}=\mu \underset{n \times 1}{(t, X, S)} d t+\underset{n \times k}{\Sigma(t, X, S)^{\prime}} \underset{k \times 1}{d W}, \\
& S\left(t_{0}\right)=S_{0},
\end{aligned}
$$

where the set of risk sources is the same we have used for the state variables. This hypothesis is not restrictive because thanks to the matrices $g$ and $\Sigma$ we can model a lot of different situations.

On the financial market there exists a riskless asset whose price $(G)$ follows the differential equation:

$$
\begin{aligned}
d G & =r(X, t) G d t, \\
G\left(t_{0}\right) & =G_{0},
\end{aligned}
$$

where $r(X, t)$ is the nominal risk-free interest rate which is supposed to depend on the state variables $X$.

\footnotetext{
${ }^{2}$ This condition can be imposed without loss of generality because a set of independent Wiener processes can always be transformed into a set of correlated Wiener processes thanks to the Cholesky decomposition.
} 


\subsection{The background risk}

We suppose that the investor is subjected to the so called background risks which can be identified with two different sets of several economic variables $\left(L \in \mathbb{R}^{l \times 1}\right.$ and $P \in \mathbb{R}^{p \times 1}$ ). In particular, we suppose that the first set of background variables affects the wealth level, while the second set affects the growth rate of investor's wealth. In other words, if $R$ is the investor's wealth, then the influence of $L$ and $P$ on the dynamic behaviour of $d R$ is given by $d L$ and $R \sum_{i=1}^{p} \frac{d P_{i}}{P_{i}}$ respectively (for more details about this distinction see the next paragraph).

Hereafter, we will refer to $L$ as the level background risk and to $P$ as the ratio background risk. Both sets of variables can be represented as diffusion processes according to the following differential equations:

$$
\begin{aligned}
& \left.\underset{l \times 1}{d L}=\mu_{L} \underset{l \times 1}{(X, L, S, t)} d t+\Lambda \underset{l \times k}{(X, L, S, t}\right)_{k \times 1}^{\prime} \underset{k \times 1}{W}, \\
& L\left(t_{0}\right)=L_{0},
\end{aligned}
$$

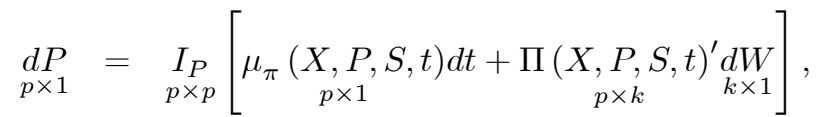

$$
\begin{aligned}
& P\left(t_{0}\right)=P_{0},
\end{aligned}
$$

where $d W$ is the same set of risk sources we have for both asset prices and state variables, and $I_{P} \in \mathbb{R}^{p \times p}$ is a diagonal matrix containing the elements of vector $P$.

We underline that the processes representing the background risks can be specified in order to consider a lot of economic problems. The following list is just an example of the variables which can be found into vectors $L$ and $P$ :

1. the inflation rate (in vector $P$ ): on the financial market we always consider nominal prices, but we should also take into account the inflation risk. If we put in the vector $P$ the stochastic process describing the behaviour of consumption prices, then we can consider the problem of maximizing the investor's real wealth (see Cox, Ingersoll, and Ross, 1985);

2. the process of wages (in vector $L$ ): we suppose to increase the investor's wealth thanks to his salary or a percentage of it (for an application see Franke, Peterson, and Stapleton, 2001). This case is useful for studying the optimal portfolio composition in the case of pension funds where the investor must contribute a percentage of his salary to the fund (for a solution with deterministic salaries see Boulier, Huang, and Taillard, 2000);

3 . in the case of an insurer, the vector $L$ can contain the present value of indemnities insurer must pay to policy holders (see Young and Zariphopoulou, 2000);

4. for a private investor the vector $L$ could contain the present value of his liabilities; 
5. if an investor holds an internationally diversified portfolio, then the vector $P$ can contain the exchange rate affecting the wealth growth rate in the same way inflation does.

\subsection{The investor's wealth}

After what we have presented in the previous subsections, the market structure can be represented in the following way:

$$
\begin{aligned}
& \left\{\begin{array}{l}
\underset{s \times 1}{d X}=f(t, X) d t+g(t, X)^{\prime} \underset{s \times 1}{d \times 1}, \\
d S=\mu(t, X, S) d t+\underset{n \times 1}{\Sigma(t, X, S)^{\prime}} \underset{k \times 1}{d W}, \\
{ }_{n \times 1}= \\
d G=G r(t, X) d t,
\end{array}\right. \\
& \underset{l \times 1}{d L}=\mu_{L}(t, \underset{l \times 1}{L}, S, X) d t+\Lambda \underset{l \times k}{(t, L, S, X)^{\prime}} \underset{k \times 1}{d W},
\end{aligned}
$$

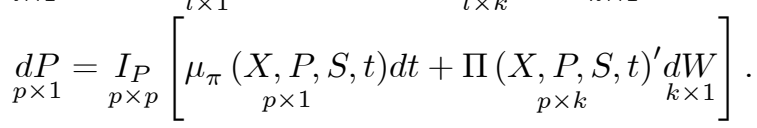

If we indicate with $\theta(t) \in \mathbb{R}^{n \times 1}$ and $\theta_{G}(t) \in \mathbb{R}$ the number of stocks bought (or sold) and the quantity of riskless asset bought (or sold) respectively, then the investor's budget constraint can be represented as:

$$
R_{m}=\theta(t)^{\prime} S+\theta_{G}(t) G+u(t)^{\prime} L,
$$

where $u(t) \in \mathbb{R}^{l \times 1}$ is the vector containing the parameters indicating in which proportion the elements of vector $L$ affect the wealth level. For instance, we could suppose that one element of $L$ is the wage process. In this case, if the investor signs a contract with a pension fund for a "definition contribution plan", then he must attribute to the fund a percentage of his wage. Thus, the vector $u(t)$ will contain an element indicating which is the percentage of investor's salary which must be paid into the fund.

After differentiating the budget constraint (6) we obtain:

$$
d R_{m}=\theta(t)^{\prime} d S+\theta_{G}(t) d G+u(t)^{\prime} d L .
$$

Nevertheless, even if the amount of money which can be invested is given by the constraint (6), we suppose investor wants to maximize the expected utility of his terminal wealth also considering that there exist some risks (for instance inflation and foreign exchange risks) affecting the growth rate of his wealth even if they do not affect the amount of money which can be invested.

Let us consider an investor having a nominal wealth equal to $R_{m}$. Even if he must invest the amount $R_{m}$, he wants to maximize the terminal utility of his real wealth $(R)$, whose growth rate can be approximated by the difference between the growth rate of nominal wealth and the growth rate of consumption prices. Nevertheless, here we do not use this approximation and we study the 
exact behaviour of investor's real wealth. If we indicate with $\phi_{m}$ the "nominal" return on investor's wealth, with $\phi$ the "real" return and with $\pi$ the inflation rate, then the following equation must hold:

$$
R \phi=R_{m}\left(\phi_{m}-\pi\right),
$$

which means that the return on the real wealth must equate the return on the nominal wealth reduced by the loss due to the increase in the price level. By definition it must be true that:

$$
\phi \equiv \frac{d R}{R}, \quad \phi_{m} \equiv \frac{d R_{m}}{R_{m}}, \quad \pi \equiv \frac{d p}{p},
$$

and so, we can write:

$$
d R=d R_{m}-R_{m} \frac{d p}{p}
$$

When we have a vector $P \in \mathbb{R}^{p \times 1}$ of risks behaving like inflation, and when these risks affect the investor's wealth through the elements of a vector $v(t) \in$ $\mathbb{R}^{p \times 1}$, then we can write the previous equation in the following way:

$$
d R=d R_{m}+R_{m} v(t)^{\prime} I_{P}^{-1} d P,
$$

where for the inflation rate, the corresponding element of $v(t)$ should be equal to -1 . Hereafter, we will not indicate the dependence of vectors $\theta, \theta_{G}, v$, and $u$ of time.

Finally, if we substitute for the differentials in the market structure (5) and for the value of $R_{m}$ given in (6) we can write:

$$
\begin{aligned}
d R= & \left(\theta^{\prime}\left(\mu+S v^{\prime} \mu_{\pi}\right)+\theta_{G}\left(G r+G v^{\prime} \mu_{\pi}\right)+u^{\prime}\left(\mu_{L}+L v^{\prime} \mu_{\pi}\right)\right) d t+ \\
& +\left(\theta^{\prime}\left(\Sigma^{\prime}+S v^{\prime} \Pi^{\prime}\right)+\theta_{G} G v^{\prime} \Pi^{\prime}+u^{\prime}\left(\Lambda^{\prime}+L v^{\prime} \Pi^{\prime}\right)\right) d W
\end{aligned}
$$

We underline that in the model where inflation is not considered, the control variable $\theta_{G}$ does not appear in the diffusion term of the wealth differential equation because the riskless asset does not contain any diffusion term. Instead, in this case, also the riskless asset is affected by the inflation diffusion term because all returns are considered in real terms.

For the sake of simplicity, we prefer to consider the following variable change:

$$
\begin{aligned}
\underset{(n+1) \times 1}{w} & =\left[\begin{array}{c}
\theta \\
\theta_{G}
\end{array}\right], \\
\underset{(n+1) \times 1}{M} & =\left[\begin{array}{c}
\mu+S v^{\prime} \mu_{\pi} \\
G r+G v^{\prime} \mu_{\pi}
\end{array}\right], \\
\underset{(n+1) \times k}{\Gamma^{\prime}} & =\left[\begin{array}{c}
\Sigma^{\prime}+S v^{\prime} \Pi^{\prime} \\
G v^{\prime} \Pi^{\prime}
\end{array}\right],
\end{aligned}
$$

and, thus, we can write the wealth differential equation as:

$$
d R=\left(w^{\prime} M+u^{\prime}\left(\mu_{L}+L v^{\prime} \mu_{\pi}\right)\right) d t+\left(w^{\prime} \Gamma^{\prime}+u^{\prime}\left(\Lambda^{\prime}+L v^{\prime} \Pi^{\prime}\right)\right) d W .
$$


With respect to the usual approach, in this differential equation we lack the term containing the wealth level. ${ }^{3}$ This characteristic comes from the following consideration: the investor is not interested in the level of his "nominal" wealth $R_{m}$, and furthermore, he cannot invest his "real" wealth $R$ because this is just a fictitious index (only nominal wealth can be actually invested). We recall that inflation is the opportunity cost of investing in financial (and not real) assets. Thus, in this work, we show how to choose the optimal investment on the financial market by considering also the investment opportunity in the real market.

\section{The optimal portfolio}

Under the market structure (5) and the evolution of investor's wealth given in Equation (7), the optimization problem for an investor maximizing the expected utility of his terminal wealth, can be written as follows:

$$
\left\{\begin{array}{l}
\max _{w} \mathbb{E}_{t_{0}}[K(R(H))] \\
d\left[\begin{array}{l}
z \\
R
\end{array}\right]=\left[\begin{array}{c}
\mu_{z} \\
w^{\prime} M+u^{\prime}\left(\mu_{L}+L v^{\prime} \mu_{\pi}\right)
\end{array}\right] d t+\left[\begin{array}{c}
\Omega^{\prime} \\
w^{\prime} \Gamma^{\prime}+u^{\prime}\left(\Lambda^{\prime}+L v^{\prime} \Pi^{\prime}\right)
\end{array}\right] d W \\
z\left(t_{0}\right)=z_{0}, R\left(t_{0}\right)=R_{0}, \quad \forall t_{0} \leq t \leq H
\end{array}\right.
$$

where:

$$
\begin{aligned}
\underset{(s+n+1+l+p) \times 1}{z} & \equiv\left[\begin{array}{lllll}
X^{\prime} & S^{\prime} & G & L^{\prime} & P^{\prime}
\end{array}\right]^{\prime}, \\
\underset{(s+n+1+l+p) \times 1}{\mu_{z}} & \equiv\left[\begin{array}{lllll}
f^{\prime} & \mu^{\prime} & G r & \mu_{L}^{\prime} & \mu_{\pi}^{\prime} I_{P}
\end{array}\right]^{\prime}, \\
\underset{k \times(s+n+1+l+p)}{\Omega} & \equiv\left[\begin{array}{lllll}
g & \Sigma & 0 & \Lambda & \Pi I_{P}
\end{array}\right],
\end{aligned}
$$

and $K(R)$ is an increasing and concave function, while $H$ is the investor's time horizon. The vector $z$ contains all the state variables but the wealth. Hereafter, we indicate with $\mathbf{0}$ a vector of suitable dimension containing only zeros.

We can write Problem (8) without considering the positivity constraint on wealth $\left(R(t) \geq 0, \forall t \in\left[t_{0}, H\right]\right)$ because Cox and Huang (1989) show that the rules derived without any constraint are asymptotically valid as wealth becomes large. Because we consider the case of an institutioinal investor, then the hypothesis of a large amount of wealth is not unlikely.

\footnotetext{
${ }^{3}$ Without any background risk and under market structure (5), the wealth differential equation should be written as follows:

$$
d R=\left(R r+\theta^{\prime}(\mu-r S)\right) d t+\theta^{\prime} \Sigma^{\prime} d W .
$$
}


From Problem (8) we have the following Hamiltonian:

$$
\begin{aligned}
\mathcal{H}= & \mu_{z}^{\prime} J_{z}+J_{R}\left(w^{\prime} M+u^{\prime}\left(\mu_{L}+L v^{\prime} \mu_{\pi}\right)\right)+\frac{1}{2} \operatorname{tr}\left(\Omega^{\prime} \Omega J_{z z}\right)+ \\
& +\left(w^{\prime} \Gamma^{\prime}+u^{\prime}\left(\Lambda^{\prime}+L v^{\prime} \Pi^{\prime}\right)\right) \Omega J_{z R}+\frac{1}{2} J_{R R}\left(w^{\prime} \Gamma^{\prime} \Gamma w+\right. \\
& \left.+u^{\prime}\left(\Lambda^{\prime}+L v^{\prime} \Pi^{\prime}\right)\left(\Lambda+\Pi v L^{\prime}\right) u+2 w^{\prime} \Gamma^{\prime}\left(\Lambda+\Pi v L^{\prime}\right) u\right),
\end{aligned}
$$

where $J(R, z, t)$ is the value function solving the Hamilton-Jacobi-Bellman partial differential equation (see Section 3.1), verifying:

$$
J(R, z, t)=\sup _{w} \mathbb{E}_{t}[K(R(H))],
$$

and the subscripts on $J$ indicate the partial derivatives.

The system of first order conditions on $\mathcal{H}$ is: ${ }^{4}$

$$
\frac{\partial \mathcal{H}}{\partial w}=J_{R} M+\Gamma^{\prime} \Omega J_{z R}+J_{R R}\left(\Gamma^{\prime} \Gamma w+\Gamma^{\prime}\left(\Lambda+\Pi v L^{\prime}\right) u\right)=0,
$$

from which we obtain the optimal portfolio composition:

$$
w^{*}=\underbrace{-\left(\Gamma^{\prime} \Gamma\right)^{-1} \Gamma^{\prime}\left(\Lambda+\Pi v L^{\prime}\right) u}_{w_{(1)}^{*}} \underbrace{-\frac{J_{R}}{J_{R R}}\left(\Gamma^{\prime} \Gamma\right)^{-1} M-\frac{1}{J_{R R}}\left(\Gamma^{\prime} \Gamma\right)^{-1} \Gamma^{\prime} \Omega J_{z R}}_{w_{(2)}^{*}} .
$$

For having a unique solution to the optimal portfolio problem we have to guarantee that the matrix $\Gamma^{\prime} \Gamma \in \mathbb{R}^{(n+1) \times(n+1)}$ is invertible. This condition is satisfied if $\Gamma^{\prime} \in \mathbb{R}^{(n+1) \times k}$ has rank equal to $n+1$ and $n+1 \leq k$. Actually, due to the ratio background risk, the riskless asset becomes a risky asset, acquiring the diffusion term of the ratio background variables. Thus, the completeness must be defined on $n+1$ assets and no more on $n$ assets. During the following work we will define a complete market as the market where the matrix $\Gamma$ is invertible (that is to say it is a square $(n+1) \times(n+1)$ matrix and its rank is maximum).

Thus, we can state the following result:

Proposition 3 Under market structure (5), the portfolio composition maximizing the investor's terminal wealth (thus solving problem (8)) is formed by three components: (i) a preference free part $\left(w_{(1)}^{*}\right)$ depending only on the diffusion terms of assets and background variables, (ii) a part $\left(w_{(2)}^{*}\right)$ proportional to both the portfolio Sharpe ratio and the inverse of Arrow-Pratt risk aversion index, and (iii) a part $\left(w_{(3)}^{*}\right)$ depending on the state variable parameters.

${ }^{4}$ The second order conditions hold if the Hessian matrix of $\mathcal{H}$ :

$$
\frac{\partial \mathcal{H}}{\partial w^{\prime} \partial w}=J_{R R} \Gamma^{\prime} \Gamma
$$

is negative definite. Because $\Gamma^{\prime} \Gamma$ is a quadratic form it is always positive definite and so the second order conditions are satisfied if and only if $J_{R R}<0$ that is if the value function is concave in $R$. The reader is referred to Stockey and Lucas (1989) for the assumptions that must hold on the function $K(R)$ for having a strictly concave value function. 
The preference free portfolio component has an important characteristic: it minimizes the wealth diffusion variance. In fact, from Equation (7) we can see that the variance of the diffusion part of investor's wealth is given by:

$$
w^{\prime} \Gamma^{\prime} \Gamma w+u^{\prime}\left(\Lambda^{\prime}+L v^{\prime} \Pi^{\prime}\right)\left(\Lambda+\Pi v L^{\prime}\right) u+2 w^{\prime} \Gamma^{\prime}\left(\Lambda+\Pi v L^{\prime}\right) u,
$$

from which we immediately see that: ${ }^{5}$

Proposition 4 The preference-free component $\left(w_{(1)}^{*}\right)$ of optimal portfolio (solving problem (8)) minimizes the wealth diffusion variance.

For the second part, we just outline that $w_{(2)}^{*}$ increases if the risk premium increases and decreases if the risk aversion or the asset variance increase. From this point of view, we can argue that this component of the optimal portfolio has just a speculative role.

The third part $w_{(3)}^{*}$ is the only optimal portfolio component explicitly depending on the diffusion terms of the state variables. Thus, while $w_{(1)}^{*}$ covers the investor from the risk "outside" the financial market (the so called background risk), $w_{(3)}^{*}$ covers the investor also from the risk "inside" the financial market. We will investigate the precise role of this component after computing the functional form of the value function.

\subsection{The value function}

For studying the exact role of the portfolio components we have called $w_{(2)}^{*}$ and $w_{(3)}^{*}$ (see Equation (10)), we need to compute the value function $J(R, z, t)$. By substituting the optimal value of $w$ into the Hamiltonian (9) we have:

$$
\begin{aligned}
\mathcal{H}^{*}= & \mu_{z}^{\prime} J_{z}-J_{R} u^{\prime}\left(\Lambda^{\prime}+L v^{\prime} \Pi^{\prime}\right) \Gamma\left(\Gamma^{\prime} \Gamma\right)^{-1} M+J_{R} u^{\prime}\left(\mu_{L}+L v^{\prime} \mu_{\pi}\right)+ \\
& -\frac{1}{2} \frac{J_{R}^{2}}{J_{R R}} M^{\prime}\left(\Gamma^{\prime} \Gamma\right)^{-1} M-\frac{J_{R}}{J_{R R}} M^{\prime}\left(\Gamma^{\prime} \Gamma\right)^{-1} \Gamma^{\prime} \Omega J_{z R}+\frac{1}{2} \operatorname{tr}\left(\Omega^{\prime} \Omega J_{z z}\right)+ \\
& +u^{\prime}\left(\Lambda^{\prime}+L v^{\prime} \Pi^{\prime}\right)\left(I-\Gamma\left(\Gamma^{\prime} \Gamma\right)^{-1} \Gamma^{\prime}\right) \Omega J_{z R}+ \\
& -\frac{1}{2} \frac{1}{J_{R R}} J_{z R}^{\prime} \Omega^{\prime} \Gamma\left(\Gamma^{\prime} \Gamma\right)^{-1} \Gamma^{\prime} \Omega J_{z R}+ \\
& +\frac{1}{2} J_{R R} u^{\prime}\left(\Lambda^{\prime}+L v^{\prime} \Pi^{\prime}\right)\left(I-\Gamma\left(\Gamma^{\prime} \Gamma\right)^{-1} \Gamma^{\prime}\right)\left(\Lambda+\Pi v L^{\prime}\right) u .
\end{aligned}
$$

${ }^{5}$ We underline that the second derivative of this term with respect to $w$ is:

$2 \Gamma^{\prime} \Gamma$,

which is always positive definite because $\Gamma^{\prime} \Gamma$ is a quadratic form. 
From this equation we can formulate the PDE whose solution is the value function. This PDE is called the Hamilton-Jacobi-Bellman equation (hereafter HJB) and it can be written as follows:

$$
\left\{\begin{array}{r}
J_{t}+\mathcal{H}^{*}=0 \\
J(H, R, z)=K(R(H))
\end{array}\right.
$$

One of the most common way to solve this kind of PDE is to try a separability condition. In the literature (since Merton, 1969, 1971), a separability by bnbproduct is generally found. Here, we suppose that the value function $J(z, R, t)$ is separable by product in wealth and in the other state variables according to the following form: $J(z, R, t)=U(R) e^{h(z, t)}$. After substituting this functional form into the HJB equation (11) and dividing by $J$ we obtain:

$$
\begin{aligned}
0= & h_{t}+\mu_{z}^{\prime} h_{z}-\frac{U_{R}}{U} u^{\prime}\left(\Lambda^{\prime}+L v^{\prime} \Pi^{\prime}\right) \Gamma\left(\Gamma^{\prime} \Gamma\right)^{-1} M+\frac{U_{R}}{U} u^{\prime}\left(\mu_{L}+L v^{\prime} \mu_{\pi}\right)+(12) \\
& -\frac{1}{2} \frac{U_{R}^{2}}{U_{R R} U} M^{\prime}\left(\Gamma^{\prime} \Gamma\right)^{-1} M-\frac{U_{R}^{2}}{U_{R R} U^{\prime}} M^{\prime}\left(\Gamma^{\prime} \Gamma\right)^{-1} \Gamma^{\prime} \Omega h_{z}+ \\
& +\frac{1}{2} \operatorname{tr}\left(\Omega^{\prime} \Omega\left(h_{z z}+h_{z} h_{z}^{\prime}\right)\right)+\frac{U_{R}}{U} u^{\prime}\left(\Lambda^{\prime}+L v^{\prime} \Pi^{\prime}\right)\left(I-\Gamma\left(\Gamma^{\prime} \Gamma\right)^{-1} \Gamma^{\prime}\right) \Omega h_{z}+ \\
& -\frac{1}{2} \frac{U_{R}^{2}}{U_{R R} U} h_{z}^{\prime} \Omega^{\prime} \Gamma\left(\Gamma^{\prime} \Gamma\right)^{-1} \Gamma^{\prime} \Omega h_{z}+ \\
& +\frac{1}{2} \frac{U_{R R}}{U} u^{\prime}\left(\Lambda^{\prime}+L v^{\prime} \Pi^{\prime}\right)\left(I-\Gamma\left(\Gamma^{\prime} \Gamma\right)^{-1} \Gamma^{\prime}\right)\left(\Lambda+\Pi v L^{\prime}\right) u .
\end{aligned}
$$

The model is consistent if and only if all the ratios containing functions of $R$ must be constant with respect to $R$. The only function satisfying all these conditions is the exponential function of the form $U(R)=\alpha e^{\gamma+\beta R}$. Accordingly, the boundary condition can be written in the following way: $U(R) e^{h(z, H)}=$ $K(R(H))$. Nevertheless, because of the separability condition, we can also write: $U(R)=K(R)$ and the boundary condition becomes: $h(z, H)=0$.

Thus, we can write:

Proposition 5 Under market structure (5) the value function is separable by product in wealth and in the other state variables if and only if the investor maximizes an exponential utility function of the form $K(R)=\alpha e^{\gamma+\beta R}$.

We just underline that we want the value function to be increasing and concave in wealth. This means that the parameters $\alpha$ and $\beta$ must be such that: $\alpha, \beta<0$, while $\gamma \in \mathbb{R}$.

If we substitute for the function $U(R)=\alpha e^{\gamma+\beta R}$ into Equation (12) we obtain:

$\left\{\begin{array}{r}h_{t}+a(z, t)^{\prime} h_{z}+b(z, t)+\frac{1}{2} \operatorname{tr}\left(\Omega^{\prime} \Omega h_{z z}\right)+\frac{1}{2} h_{z}^{\prime} \Omega^{\prime}\left(I-\Gamma\left(\Gamma^{\prime} \Gamma\right)^{-1} \Gamma^{\prime}\right) \Omega h_{z}=0 \\ h(z, H)=0 .\end{array}\right.$ 
where:

$$
\begin{aligned}
a(z, t)^{\prime} \equiv & \mu_{z}^{\prime}-M^{\prime}\left(\Gamma^{\prime} \Gamma\right)^{-1} \Gamma^{\prime} \Omega+\beta u^{\prime}\left(\Lambda^{\prime}+L v^{\prime} \Pi^{\prime}\right)\left(I-\Gamma\left(\Gamma^{\prime} \Gamma\right)^{-1} \Gamma^{\prime}\right) \Omega, \\
b(z, t) \equiv & \beta u^{\prime}\left(\mu_{L}+L v^{\prime} \mu_{\pi}\right)-\beta u^{\prime}\left(\Lambda^{\prime}+L v^{\prime} \Pi^{\prime}\right) \Gamma\left(\Gamma^{\prime} \Gamma\right)^{-1} M-\frac{1}{2} M^{\prime}\left(\Gamma^{\prime} \Gamma\right)^{-1} M+ \\
& +\frac{1}{2} \beta^{2} u^{\prime}\left(\Lambda^{\prime}+L v^{\prime} \Pi^{\prime}\right)\left(I-\Gamma\left(\Gamma^{\prime} \Gamma\right)^{-1} \Gamma^{\prime}\right)\left(\Lambda+\Pi v L^{\prime}\right) u .
\end{aligned}
$$

By using the exponential utility function and the separability condition, we can write the optimal portfolio composition in the following way:

$$
w^{*}=-\left(\Gamma^{\prime} \Gamma\right)^{-1} \Gamma^{\prime}\left(\Lambda+\Pi v L^{\prime}\right) u-\frac{1}{\beta}\left(\Gamma^{\prime} \Gamma\right)^{-1} M-\frac{1}{\beta}\left(\Gamma^{\prime} \Gamma\right)^{-1} \Gamma^{\prime} \Omega h_{z},
$$

and, for finding a close form solution we have to solve the HJB equation in order to find the form of function $h(z, t)$. In the following subsections we study how to solve Equation (13) in both a complete and an incomplete market.

\subsection{The case of a complete market: an exact solution}

Because of the insertion of the so called ratio background risk, the first definition of a complete market, given on the matrix $\Sigma$ of the diffusion terms of the risky assets, must be restate by using the matrix $\Gamma$. Actually, under a ratio background risk the riskless asset looses its lack of risk and it acquires the diffusion term of the background variable. Thus, the completeness of the market must be specified on $n+1$ assets and we can say that the market is complete if the matrix $\Gamma$ can be inverted.

If $\Gamma^{-1}$ does exist, then the term $\left(I-\Gamma\left(\Gamma^{\prime} \Gamma\right)^{-1} \Gamma^{\prime}\right)$ in Equation (13) vanishes and we obtain a partial differential equation which can be solved thanks to the Feynman-Kac theorem. ${ }^{6}$ Thus, we can state:

Proposition 6 Under market structure (5), if the market is complete for $n+1$ risky assets, then the portfolio composition maximizing the investor's terminal exponential utility function $\left(K(R)=\alpha e^{\gamma+\beta R}\right)$ is as follows:

$$
w^{*}=-\Gamma^{-1}\left(\Lambda+\Pi v L^{\prime}\right) u-\frac{1}{\beta}\left(\Gamma^{\prime} \Gamma\right)^{-1} M-\frac{1}{\beta} \Gamma^{-1} \Omega \int_{t}^{H} \frac{\partial}{\partial z} \mathbb{E}_{t}\left[b\left(Z_{s}, s\right)\right] d s,
$$

where:

$$
\begin{aligned}
d Z_{s} & =a\left(Z_{s}, t\right) d s+\Omega\left(Z_{s}, s\right)^{\prime} d W \\
Z_{t} & =z \\
a(z, t)^{\prime} & \equiv \mu_{z}^{\prime}-M^{\prime} \Gamma^{-1} \Omega \\
b(z, t) & \equiv \beta u^{\prime}\left(\mu_{L}+L v^{\prime} \mu_{\pi}\right)-\beta u^{\prime}\left(\Lambda^{\prime}+L v^{\prime} \Pi^{\prime}\right) \Gamma^{\prime-1} M-\frac{1}{2} M^{\prime}\left(\Gamma^{\prime} \Gamma\right)^{-1} M .
\end{aligned}
$$

\footnotetext{
${ }^{6}$ For a complete exposition of the Feynman-Kac theorem the reader is referred to Duffie (1996), Björk (1998) and Øksendal (2000).
} 
We can see that the only component of optimal portfolio explicitly depending on the investor's horizon $H$ is the third one which hedges the portfolio against the state variable risks and, in particular, against the background risks.

For finding an exact solution, we must consider that state variables following a modified stochastic process with respect to the original one. Nevertheless, since the diffusion term $\Omega$ has not changed, then we can use the Girsanov theorem $^{7}$ for finding a suitable probability measure in order to switch from one process to the other.

This result is important because it shows the possibility to obtain an exact close form solution in the case where all the risks affecting the portfolio composition can be represented by diffusion processes. We underline that Proposition 6 is obtained without specifying any functional form for the coefficients of the stochastic processes implied in the solution. In fact, the exact solution already found in the literature by Kim and Omberg (1996), Wachter (1998), Boulier, Huang, and Taillard (2001), and Deelstra, Grasselli, and Koehl (2001) can be obtained under the hypothesis that there exists only one state variable following a Vasicek (1977) process or a Cox, Ingersoll, and Ross (1985) process.

\subsection{The case of an incomplete market: an approximated solution}

Unfortunately, if the market is not complete and we cannot inverse the matrix $\Gamma$, then we are not able to apply the Feynman-Kac theorem to Equation (13) because of the term containing $h_{z} h_{z}^{\prime}$. In order to apply the theorem, we should have only the term $h_{z z}$. If we impose $h_{z}$ to be zero, then also $h_{z z}$ must be zero and a solution to (13) can be found only if the term $b(z, t)$ is independent of $z$. This is a too particular case for being interesting.

We could choose a kind of functional form for $h(z, t)$ such that:

$$
\frac{1}{2} h_{z}^{\prime} \Omega^{\prime}\left(I-\Gamma\left(\Gamma^{\prime} \Gamma\right)^{-1} \Gamma^{\prime}\right) \Omega h_{z}=h,
$$

and also in this case we could apply the Feynman-Kac theorem. Nevertheless, if $\Omega^{\prime}\left(I-\Gamma\left(\Gamma^{\prime} \Gamma\right)^{-1} \Gamma^{\prime}\right) \Omega$ does not depend on $z$ then this function must be a quadratic polynomial of the form:

$$
\begin{aligned}
h(z, t)= & \frac{1}{2} B(t)^{\prime} \Omega^{\prime}\left(I-\Gamma\left(\Gamma^{\prime} \Gamma\right)^{-1} \Gamma^{\prime}\right) \Omega B(t)+B(t)^{\prime} z+ \\
& +\frac{1}{2} z^{\prime}\left(\Omega^{\prime}\left(I-\Gamma\left(\Gamma^{\prime} \Gamma\right)^{-1} \Gamma^{\prime}\right) \Omega\right)^{-1} z
\end{aligned}
$$

where only $B(t)$ can be arbitrarily chosen. Nevertheless, we do not want to impose such a restrictive condition. If $\Omega^{\prime}\left(I-\Gamma\left(\Gamma^{\prime} \Gamma\right)^{-1} \Gamma^{\prime}\right) \Omega$ actually depends

\footnotetext{
${ }^{7}$ For a complete exposition of the Girsanov theorem the reader is referred to Karatzas and Shreve (1998), and Øksendal (2000).
} 
on $z$ then $h(z, t)$ must be an integral function in $z$ which is very difficult to manage.

Instead, we prefer to search for a function satisfying $h_{z z}=h_{z} h_{z}^{\prime}$. After solving this differential equation, we find that $h(z, t)$ must have the following form:

$$
h(z, t)=A(t)-\ln \left(B(t)^{\prime} z+D(t)\right),
$$

where $A(t), D(t) \in \mathbb{R}$, and $B(t) \in \mathbb{R}^{(s+n+1+l+p) \times 1}$ such that $B(t)^{\prime} z+D(t)>$ 0 . In this case, in fact, we have:

$$
h_{z z}=\frac{B(t) B(t)^{\prime}}{\left[B(t)^{\prime} z+D(t)\right]^{2}}=h_{z} h_{z}^{\prime} .
$$

Thus, if the function $h(z, t)$ has the form (14), then the HJB equation can be simplified as follows:

$$
\left\{\begin{aligned}
h_{t}+a(z, t)^{\prime} h_{z}+b(z, t)+\frac{1}{2} \operatorname{tr}\left[\Omega^{\prime}\left(2 I-\Gamma\left(\Gamma^{\prime} \Gamma\right)^{-1} \Gamma^{\prime}\right) \Omega h_{z z}\right] & =0 \\
h(H, z) & =0 .
\end{aligned}\right.
$$

For applying the Feynman-Kac theorem, we have to find two real numbers $x_{1}$ and $x_{2}$ such that:

$$
\left(x_{1} I-x_{2} \Gamma\left(\Gamma^{\prime} \Gamma\right)^{-1} \Gamma^{\prime}\right)^{2}=2 I-\Gamma\left(\Gamma^{\prime} \Gamma\right)^{-1} \Gamma^{\prime},
$$

from which, because $I-\Gamma\left(\Gamma^{\prime} \Gamma\right)^{-1} \Gamma^{\prime}$ is a symmetric, idempotent matrix, we easily obtain $x_{1}=\sqrt{2}$ and $x_{2}=\sqrt{2} \pm 1$. Thus, by putting:

$$
\widetilde{\Omega}^{\prime} \equiv \Omega^{\prime}\left[\sqrt{2} I-(\sqrt{2} \pm 1) \Gamma\left(\Gamma^{\prime} \Gamma\right)^{-1} \Gamma^{\prime}\right]^{\prime},
$$

we can write the HJB equation in the following way:

$$
\left\{\begin{aligned}
h_{t}+a(z, t)^{\prime} h_{z}+b(z, t)+\frac{1}{2} \operatorname{tr}\left(\widetilde{\Omega^{\prime}} \widetilde{\Omega} h_{z z}\right) & =0, \\
h(H, z) & =0 .
\end{aligned}\right.
$$

Now, we are able to apply the Feynman-Kac theorem, and the solution of the HJB equation is given by:

$$
h(z, t)=\int_{t}^{H} \mathbb{E}_{t}\left[b\left(Z_{s}, s\right)\right] d s,
$$

where the variables $Z_{s}$ follow:

$$
\begin{aligned}
d Z_{s} & =a\left(Z_{s}, t\right) d s+\widetilde{\Omega}\left(Z_{s}, s\right)^{\prime} d W \\
Z_{t} & =z .
\end{aligned}
$$

Thus, our result, can be summarized as follows: 
Proposition 7 Under market structure (5), if the market is incomplete, then the portfolio composition maximizing the investor's terminal exponential utility function $\left(K(R)=\alpha e^{\gamma+\beta R}\right)$ is given by:

$$
\begin{aligned}
w^{*}= & -\left(\Gamma^{\prime} \Gamma\right)^{-1} \Gamma^{\prime}\left(\Lambda+\Pi v L^{\prime}\right) u-\frac{1}{\beta}\left(\Gamma^{\prime} \Gamma\right)^{-1} M+ \\
& -\frac{1}{\beta}\left(\Gamma^{\prime} \Gamma\right)^{-1} \Gamma^{\prime} \Omega \int_{t}^{H} \frac{\partial}{\partial z} \mathbb{E}_{t}\left[b\left(Z_{s}, s\right)\right] d s,
\end{aligned}
$$

if and only if there exist functions $A(t), D(t) \in \mathbb{R}$, and $B(t) \in \mathbb{R}^{(s+n+1+l+p) \times 1}$ such that:

$$
\int_{t}^{H} \mathbb{E}_{t}\left[b\left(Z_{s}, s\right)\right] d s=A(t)-\ln \left(B(t)^{\prime} z+D(t)\right),
$$

where:

$$
\begin{aligned}
d Z_{s}= & a\left(Z_{s}, t\right) d s+\widetilde{\Omega}\left(Z_{s}, s\right)^{\prime} d W \\
Z_{t}= & z \\
a(z, t)^{\prime} \equiv & \mu_{z}^{\prime}-M^{\prime}\left(\Gamma^{\prime} \Gamma\right)^{-1} \Gamma^{\prime} \Omega+\beta u^{\prime}\left(\Lambda^{\prime}+L v^{\prime} \Pi^{\prime}\right)\left(I-\Gamma\left(\Gamma^{\prime} \Gamma\right)^{-1} \Gamma^{\prime}\right) \Omega, \\
b(z, t) \equiv & \beta u^{\prime}\left(\mu_{L}+L v^{\prime} \mu_{\pi}\right)-\beta u^{\prime}\left(\Lambda^{\prime}+L v^{\prime} \Pi^{\prime}\right) \Gamma\left(\Gamma^{\prime} \Gamma\right)^{-1} M-\frac{1}{2} M^{\prime}\left(\Gamma^{\prime} \Gamma\right)^{-1} M+ \\
& +\frac{1}{2} \beta^{2} u^{\prime}\left(\Lambda^{\prime}+L v^{\prime} \Pi^{\prime}\right)\left(I-\Gamma\left(\Gamma^{\prime} \Gamma\right)^{-1} \Gamma^{\prime}\right)\left(\Lambda+\Pi v L^{\prime}\right) u \\
\widetilde{\Omega}^{\prime} \equiv & \Omega^{\prime}\left[\sqrt{2} I-(\sqrt{2} \pm 1) \Gamma\left(\Gamma^{\prime} \Gamma\right)^{-1} \Gamma^{\prime}\right]^{\prime}
\end{aligned}
$$

The closed form solution for the portfolio allocation problem obtained in Proposition 7 is valid if and only if the function $h(z, t)$ has the form (14). If the function $h(z, t)$ cannot be written in this way, then our previous result cannot be applied.

Nevertheless, we can state that the result obtained in Proposition 7 is still valid as an approximation of the true result. If we develop in Taylor series the function $h(z, t)$ around a given value of $z$ (let us say $z_{0}$ ), then we obtain:

$$
\begin{aligned}
h(z, t)= & A(t)-\ln \left(B(t)^{\prime} z_{0}+D(t)\right)-\frac{B(t)^{\prime}\left(z-z_{0}\right)}{B(t)^{\prime} z_{0}+D(t)}+ \\
& +\frac{\left(z-z_{0}\right)^{\prime} B(t)^{\prime} B(t)\left(z-z_{0}\right)}{2\left(B(t)^{\prime} z_{0}+D(t)\right)^{2}}+O\left(\left\|z-z_{0}\right\|^{3}\right) .
\end{aligned}
$$

Thus, if the function $h(z, t)$ can be expressed in the form (15), that is to say as a polynomial in $z$, then our result can approximate the exact solution. We underline that Kim and Omberg (1996) find, for an incomplete market, an exact solution where the function $h(z, t)$ is a second degree polynomial in $z$. 


\subsection{The third component of optimal portfolio}

As we have already underlined, the third part of optimal portfolio is the only one depending on the investor's horizon. Thus, if we want to consider an investor with an infinite time horizon, then we must verify that the integral in Proposition 6 converges.

From our previous propositions it can be seen that if all parameters of our problem do not depend on state or background variables but depend only on time, then the derivative term $\frac{\partial}{\partial z} \mathbb{E}_{t}\left[b\left(Z_{s}, s\right)\right]$ vanishes (because $\frac{\partial}{\partial Z_{s}} b\left(Z_{s}, s\right)=$ $\mathbf{0})$.

Thus, we can state:

Proposition 8 If the coefficients of the stochastic processes in the market structure (5) depend only on time, then the third component of the optimal portfolio $\left(w_{(3)}^{*}\right)$ vanishes.

In the work by Lioui and Poncet (2000) it is shown that, if the market is complete, then the third component of the optimal portfolio is formed only by two parts, even though the number of state variables is arbitrarily large. In particular, the first part is associated with the interest rate risk and the second one with the market price of risk. Even if Lioui and Poncet use the martingale approach, here we underline that we obtain the same qualitative result.

Because the authors do not introduce any background variable, ${ }^{8}$ then we have to put in our framework $u=\mathbf{0}$. Under this hypothesis we can see from Proposition 6 that the function $h(z, t)$ is formed only by one term and, more precisely, we have:

$$
b(z, t) \equiv-\frac{1}{2} M^{\prime}\left(\Gamma^{\prime} \Gamma\right)^{-1} M
$$

but for understanding its meaning better we must recall the form of matrices $M$ and $\Gamma$. Because matrix $\left(\Gamma^{\prime} \Gamma\right)^{-1}$ does not explicitly contain the nominal riskless interest rate $r$, then we can just write a generic matrix:

$$
b(z, t) \equiv-\frac{1}{2}\left[\begin{array}{c}
\mu+S v^{\prime} \mu_{\pi} \\
G r+G v^{\prime} \mu_{\pi}
\end{array}\right]^{\prime}\left[\begin{array}{cc}
X & x \\
n \times n & n \times 1 \\
x^{\prime} & y \\
1 \times n &
\end{array}\right]\left[\begin{array}{c}
\mu+S v^{\prime} \mu_{\pi} \\
G r+G v^{\prime} \mu_{\pi}
\end{array}\right]
$$

from which we can write:

$$
\begin{aligned}
b(z, t) \equiv & -\frac{1}{2}\left(G r+G v^{\prime} \mu_{\pi}\right)^{2} y-\left(G r+G v^{\prime} \mu_{\pi}\right) x^{\prime}\left(\mu+S v^{\prime} \mu_{\pi}\right)+ \\
& -\frac{1}{2}\left(\mu^{\prime}+\mu_{\pi}^{\prime} v S^{\prime}\right) X\left(\mu+S v^{\prime} \mu_{\pi}\right) .
\end{aligned}
$$

\footnotetext{
${ }^{8}$ We outline that they define an investor who is endowed with a portfolio of discount bonds that he chooses not to trade until his investment horizon $(H)$. This hypothesis allows the authors to have a non-zero first portfolio component $w_{(1)}^{*}$.
} 
Accordingly, in our framework, we are not able to disentangle the two risks linked to the interest rate $r$ and to the market price of risk. Furthermore, if we try to distinguish the terms in $r$, then we find a second degree polynomial in $r$. This is due to the insertion of the ratio background risk. In fact, here the riskless asset becomes like the other risky assets, and the risk linked to the interest rate $r$ becomes a component of the market price of risk. Nevertheless, the qualitative result after Lioui and Poncet is preserved.

By comparing the approximated and the exact solutions presented in Propositions 7 and 6 respectively, we can see that the approximation $h_{z} h_{z}^{\prime}=h_{z z}$ implies that a positive definite matrix is added to the original term containing $h_{z z}$. In fact, the HJB equation has been written as follows:

$$
\begin{gathered}
h_{t}+a(z, t)^{\prime} h_{z}+b(z, t)+\frac{1}{2} \operatorname{tr}\left[\Omega^{\prime} \Omega h_{z z}\right]+ \\
+\frac{1}{2} h_{z}^{\prime}\left(\Omega^{\prime}\left(I-\Gamma\left(\Gamma^{\prime} \Gamma\right)^{-1} \Gamma^{\prime}\right) \Omega\right) h_{z}=0 .
\end{gathered}
$$

Accordingly, after putting $h_{z} h_{z}^{\prime}=h_{z z}$, the matrix $\Omega^{\prime}\left(I-\Gamma\left(\Gamma^{\prime} \Gamma\right)^{-1} \Gamma^{\prime}\right) \Omega$ is added to the matrix $\Omega^{\prime} \Omega$. We underline that the coefficient of $h_{z z}$ is the diffusion term of the stochastic process according to which the variables $z$ must behave under the Feynman-Kac theorem. Since the first matrix is positive semi-definite, this means that we are going to add some risks to the original ones and so, in the approximated solution we have presented in Proposition 7, the optimal portfolio third component always overestimates the exact solution. Thus, we can conclude:

Corollary 9 The approximated solution for the third optimal portfolio component $\left(w_{(3)}^{*}\right)$ presented in Proposition 7 systematically overestimates the exact solution.

The future work will concentrate on the measure of this overestimation. We have already seen that the error is negligible if the value function can be written as an exponential of a polynomial in the state variables. Actually, in order to offer a good approximation even in the other cases, we need some more considerations.

\section{Conclusion}

In this paper we have analysed the asset allocation problem for an investor maximizing the expected value of his terminal exponential utility function. The investor faces an economic environment with stochastic investment opportunities and both a complete and an incomplete financial market are considered. Furthermore, he must cope with two sets of background risks: one called level 
background risk and affecting the level of his wealth (as salaries), the other one called ratio background risk and affecting the wealth growth rate (as inflation).

The optimal portfolio is formed by three components: (i) a preference free part depending only on the diffusion terms of assets and background variables, (ii) a part proportional to both the portfolio Sharpe ratio and the inverse of Arrow-Pratt risk aversion index, and (iii) a part depending on all the state variable parameters.

We show that the preference-free component minimizes the diffusion variance of investor's wealth.

For understanding the role of the third component it is necessary to explicitly compute the value function solving the dynamic stochastic optimization problem. With respect to the most common approach used in the literature, the insertion of the ratio background risk allows us to find an exact solution for the optimal portfolio problem when the financial market is complete. Furthermore, when the market is incomplete, we suggest a method for finding an approximated solution.

We underline that all our results, contrary to the exact solutions found in the literature, are obtained without specifying any particular functional form for the stochastic variables involved in the problem. Thus, the solutions we are able to find are very general and can be applied to several economic problems.

\section{References}

[1] Barberis, N. (2000). Investigating for the long run when returns are predictable. Journal of Finance, 55, 225-264.

[2] Bellamy, N. (2001). Wealth optimization in an incomplete market driven by a jump-diffusion process. Journal of Mathematical Economics, 35, 259-287.

[3] Björk, T. (1998). Arbitrage Theory in Continuous Time. Oxford University Press, New York.

[4] Blake, D. (1998). Pension schemes as options on pension fund assets: implications for pension fund management. Insurance: Mathematics and Economics, 23, 263-286.

[5] Blake, D., Cairns, A., Dowd, K. (1998). Pension Metrics: Stochastic Pension Plan Design and Value-At-Risk during the Accumulation Phase. Working Paper, BSI Gamma Foundation.

[6] Boulier, J.-F., Huang, S. J., Taillard, G. (2001). Optimal Management Under Stochastic Interest. Insurance: Mathematics and Economics, 28, 173-189.

[7] Campbell, J. Y. (2000). Asset Pricing at the Millennium. The Journal of Finance, 55, 1515-1567. 
[8] Campbell, J. Y., Viceira, L. M. (1999). Consumption and Portfolio Decisions When Expected Returns are Time Varying. Quarterly Journal of Economics, 114, 433-495.

[9] Chacko, G., Viceira, L. M. (1999). Dynamic consumption and portfolio choice with stochastic volatility in incomplete markets. Working Paper, Harvard University.

[10] Cox, J. C., Huang, C. F. (1989). Optimal consumption and portfolio policies when asset prices follow a diffusion process. Journal of Economic Theory, 49, 33-83.

[11] Cox, J. C., Huang, C. F. (1991). A variational problem arising in financial economics. Journal of Mathematical Economics, 20, 465-487.

[12] Cox, J. C., Ingersoll, J. E. Jr., Ross, S. A. (1985). A Theory of the Term Structure of Interest Rates. Econometrica, 53, 385-407.

[13] Deelstra, G., Grasselli, M., Koehl, P.-F. (2001). Optimal Investment Strategies in a CIR Framework. Forthcoming in Journal of Applied Probability.

[14] Duffie, D. (1996). Dynamic Asset Pricing Theory. Second edition, Princeton University Press.

[15] Franke, G., Peterson, S., Stapleton, R. C. (2001). Intertemporal Portfolio Behaviour When Labor Income is Uncertain. SIRIF Conference on "Dynamic Portfolio Strategies", Edinburgh, May.

[16] Karatzas, I., Shreve, E. S. (1998). Methods of Mathematical Finance. Springer-Verlag, New York, NY.

[17] Kim, T. S., Omberg, E. (1996). Dynamic Nonmyopic Portfolio Behavior. The Review of Financial Studies, 9, 141-161.

[18] Kogan, L., Uppal, R. (1999). Risk Aversion and Optimal Portfolio Policies in Partial and General Equilibrium Economies. Working Paper, Wharton.

[19] Lioui, A., Poncet, P. (2000). On Optimal Portfolio Choice under Stochastic Interest Rates. Forthcoming in Journal of Economic Dynamics and Control.

[20] Merton, R. C. (1969). Lifetime Portfolio Selection under Uncertainty: the Continuous-Time Case. Review of Economics and Statistics, 51, 247-257.

[21] Merton, R. C. (1971). Optimum Consumption and Portfolio Rules in a Continuous-Time Model. Journal of Economic Theory, 3, 373-413.

[22] Merton, R. C. (1990). Continuous-Time Finance. Blackwell, Cambridge, MA.

[23] Øksendal, B. (2000). Stochastic Differential Equations - An Introduction with Applications. Fifth edition, Springer, Berlin. 
[24] Stockey, N. L., Lucas, R. E. Jr. (1989). Recursive Methods in Economic Dynamics. Harvard University Press, Cambridge, Massachusetts.

[25] Vasicek, O. (1977). An Equilibrium characterization of the Term Structure. Journal of Financial Economics, 5, 177-188.

[26] Wachter, J. A. (1998). Portfolio and Consumption Decisions Under MeanReverting Returns: An Exact Solution for Complete Markets. Working Paper, Harvard University.

[27] Young, V. R., Zariphopoulou, T. (2000). Pricing Insurance via Stochastic Control: Optimal Terminal Wealth. MIMEO.

[28] Zariphopoulou, T. (2001). A solution approach to valuation with unhedgeable risks. Finance and Stochastics, 5, 61-82. 